\title{
Operations research in neuroscience
}

\author{
Erik Kropat $^{1}$ • Ruben A. Tikidji-Hamburyan ${ }^{2}$. \\ Gerhard-Wilhelm Weber ${ }^{3}$
}

Published online: 14 September 2017

(C) Springer Science+Business Media, LLC 2017

Neuroscience is a multidisciplinary science that is concerned with the study of the structure and function of the nervous system. It comprises aspects of physiology, cellular and molecular biology, as well as behavioral and cognitive sciences. Recent technological and theoretical advances offer promising opportunities for better understanding of the nervous system at the quantitative level. New optogenetic, in vitro and in vivo multielectrode recording, neuroimaging, brain imaging, and other modern techniques provide high-quality data that can be used in elucidating the fundamental mechanisms of information processing in neural systems. The rapidly growing pool of experimental data has triggered the development of new mathematical models that can provide new insights into the functioning of the nervous system at various scales ranging from that of molecular biology to that of the organizational principles of behavior and cognition. Sophisticated analyses of intracellular signaling or dynamics in heterogeneous neural networks, conditional behavior, or connections of brain regions in decision-making lead to theoretical problems addressed by operations research.

The special issue covers a broad range of topics in the field of neuroscience that use operations research approaches and methods. The articles include, but are not limited to, the following areas of Neuroscience:

- intracellular signaling and regulation

- dynamics in heterogeneous neural networks

\footnotetext{
$\bowtie$ Erik Kropat

Erik.Kropat@gmx.de

Ruben A. Tikidji-Hamburyan rath@gwu.edu

Gerhard-Wilhelm Weber gweber@metu.edu.tr

1 Institute of Applied Informatics, University of the Bundeswehr, Munich, 85577 Neubiberg, Germany

2 School of Engineering and Applied Science, George Washington University, Washington, DC, USA

3 Institute of Applied Mathematics, Middle East Technical University, 06800 Ankara, Turkey
} 
- perceptual, cognitive and behavioral functions

- learning and memory

- spatio-temporal network analysis

- neuroimaging and functional brain imaging processing

- computational modelling and simulation

In the following, we shall give a short representation of the contents of the special issue.

Experimental records show the existence of a biological linkage between neuronal death and Golgi apparatus fragmentation. The comprehension of such a linkage should help to understand the dynamics undergoing neurological damage caused by diseases such as Alzheimer's disease or amyotrophic lateral sclerosis. The authors Eduardo Álvarez-Miranda, Hesso Farhan, Martin Luipersbeck, and Markus Sinnl propose using the bi-objective minimum cardinality bottleneck Steiner tree problem along with an ad-hoc exact algorithm to study such phenomena. The suggested algorithm is based on both Integer Programming (IP) and the Epsilon-constraint Method. A core property of the approach is that it allows an efficient IP problem formulation. The results give additional evidence supporting the hypothesis that alterations of the Golgi apparatus structure and neuronal death interact through biological mechanisms underlying the outbreak and progression of neurodegenerative diseases. Furthermore, the function of cellular response to stress as a biological linkage between those phenomena is investigated. Finally, computational results on a synthetic dataset are provided aiming to report the performance of the recommended procedure.

Operations research is increasingly used in neuroscience for diagnosing neurodegenerative diseases with computer-aided systems based on brain image analysis. Though the use of multiple biomarkers derived from Magnetic Resonance Imaging (MRI) data have proven effective in diagnosing Alzheimer's disease (AD) and mild cognitive impairment (MCI), no specific system has been a part of routine clinical practice yet. The paper by Alper Çevik, Gerhard-Wilhelm Weber, B. Murat Eyüboğlu, Kader Karlı Oğuz, and The Alzheimer's Disease Neuroimaging Initiative introduces a fully automated voxel-based procedure, VoxelMARS, for the detection of AD and MCI at early stages of progression. The performance was evaluated on a dataset of 508 MRI volumes gathered from the AD's Neuroimaging Initiative (ADNI) database. Data were transformed into a high-dimensional space through a feature extraction process, and a novel 3-step feature selection procedure was applied. The Multivariate Adaptive Regression Splines (MARS) method was used as a classifier for the first time in the field of brain MRI analysis. The results were compared to those presented in a previous study on 28 voxel-based methods with regard to their ability to separate control normal $(\mathrm{CN})$ subjects from the ones diagnosed with $\mathrm{AD}$ and MCI. The authors' method outperformed all the others in sensitivity $(83.58 \%$ in $\mathrm{AD} / \mathrm{CN}$ and $78.38 \%$ in $\mathrm{MCI} / \mathrm{CN}$ classification) with acceptable specificity values (over $85 \%$ in both cases). Moreover, the method worked for discriminating MCI patients who converted to AD in 18 months (MCIc) from non-converters (MCInc) with a sensitivity outcome better than 27 of 28 methods. Indeed, it was demonstrated that the suggested method is promising for early detection of AD.

One popular application of operations research in data analysis is used in the study of inattention detection of operators in human-machine interaction systems using an electroencephalography (EEG) signal. Most of the previous studies on inattention detection employed supervised learning approaches, but the results have a potential bias since they rely on imperfect assumptions for the acquisition of mental state labels-attention and inattention-due to the absence of standardized measure for the mental states. The authors Yerim Choi, Jonghun Park and Dongmin Shin instead follow an unsupervised learning approach, with no labeled data being needed. To address the low performance of unsupervised learning methods, attention 
duration for which an operator sustains attention from the beginning of performing a task and relevance levels between 4 attributes of EEG signal and mental states are exploited. Here, they suggest a semi-supervised inattention detection method (SID), in which attention duration and attributes-weights of EEG signal are respectively utilized as a small portion of labeled data for semi-supervised learning and weights for similarity calculation. Specifically, the Cumulative Sum (CUSUM) algorithm is applied to determine the attention duration, and the Constrained Attributes-weighting Clustering algorithm is used to estimate the attributes' weights. Using a real-world dataset, SID outperforms the compared methods, and it is expected that the adoption of SID will enhance the operators' safety.

The activator-inhibitor FitzHugh-Nagumo (FHN) equation is a reaction-diffusion equation with skew-gradient structure. The authors Bülent Karasözen, Tuğba Küçükseyhan and Murat Uzunca discretize the FHN equation by using the symmetric interior penalty discontinuous Galerkin (SIPG) method in space and the average vector field (AVF) method in time. The AVF method is a geometric integrator, i.e., it preserves the energy of the Hamiltonian systems and energy dissipation of the gradient systems. In this article, the authors show that the fully discrete energy of the FHN equation fulfills the mini-maximizer property of continuous energy for skew-gradient systems. They provide numerical results with traveling fronts and pulses for 1-dimensional, 2-coupled FHN equations and 3-coupled FHN equations with 1 activator and 2 inhibitors in skew-gradient form. So-called Turing patterns are computed for a fully discretized 2-dimensional FHN equation in the form of spots and labyrinths. Since the computation of the Turing patterns is time consuming for different parameters, the authors applied model-order reduction with Proper Orthogonal Decomposition (POD). The nonlinear term in the reduced equations is computed using Discrete Empirical Interpolation (DEIM) with SIPG discretization. Because of the local nature of the Discontinuous Galerkin (DG) method, the nonlinear terms can be computed more efficiently than for continuous finite elements. The reduced solutions are very close to the fully discretized ones. Efficiency and accuracy of the POD and POD-DEIM reduced solutions are demonstrated for labyrinth-like patterns.

For solving a sequential decision-making problem in a non-Markovian domain, standard Dynamic Programming (DP) requires a complete mathematical model, thus, it is a totally model-based approach. The contribution by Eiji Mizutani and Stuart Dreyfus, however, describes a totally model-free approach by actor-critic reinforcement learning with recurrent neural networks. The recurrent connections (or context units) in neural networks act as an implicit form of internal state (i.e., history memory) for developing sensitivity to hidden non-Markovian dependencies, rendering the process Markovian implicitly and automatically in a totally model-free fashion. Thus, the model-free recurrent-network agent neither learns transitional probabilities and associated rewards, nor by how much the state space should be enlarged so that the Markov property holds. For concreteness of exposition, the authors illustrate time-lagged path problems, where their learning agent is expected to learn a best policy that maximizes the total return, the sum of one-step transitional rewards plus special "bonus" values that depend on prior transitions or decisions. As they can obtain an optimal solution by model-based DP, this is an excellent test on the learning agent for understanding its model-free learning behavior. Such actor-critic recurrent-network learning might constitute a mechanism that animal brains use when experimentally acquiring skilled action. Given a concrete non-Markovian problem example, the goal of this paper is to show the conceptual merit of totally model-free learning with actor-critic recurrent networks, compared with classical DP (and other model-building procedures), rather than to pursue a best recurrent-network learning strategy. 
While developing models of brain functioning by using time-series data, the stationary interval of the time series should be used to model the corresponding state of the brain. Here, it is assumed that at the borders of stationarity, the brain changes its state, where a state is considered as a group of brain regions working together. If the entire nonstationary time series is used, many different brain states could be included in one single model. But it is very hard to decide on the stationary intervals for such a complicated system as the brain. In the literature, there are some methods that demonstrate their performances based on manually constructed synthetic data. Only very few results with EEG data have been presented. But, usually there is no ground truth accompanying the data to make an evaluation. In the work of V. Resoulzadeh, E.C. Erkus, T.A. Yogurt, I. Ulusoy, and S. Aykan Zergeroğlu, suitable approaches for stationary analysis were applied on visual evoked potentials (VEP) where they can approximately know the possible stationary intervals due to the properties of the experiment during which the data were recorded. Experts designed the experiment and marked the possible borders of the intervals carefully. Parameters of the methods were set automatically. The authors compared manually marked intervals with intervals detected automatically by the applied methods and evaluated the methods according to their performances in estimating the stationary intervals of EEG signals.

In their article, Mustafa Şaylı and Enes Yılmaz address a new model of neural networks related to the impulsive phenomena that are called state-dependent impulsive recurrent neural networks with time-varying and continuously distributed delays. The authors investigate sufficient conditions on the existence and uniqueness of exponentially stable antiperiodic solution for these neural networks by employing the method of Coincide Degree Theory and an appropriate Lyapunov function. Moreover, they provide an example to illustrate the effectiveness and feasibility of the theoretical results obtained.

As Guest Editors, we hope that the selected topics well display a core selection of international research coping with the emerging and complex problems of neuroscience and its associated fields in medicine by using the results and methods of operations research. We are very thankful to the Annals of Operations Research and the publishing house of Springer for the honor of hosting this special issue as a pioneering scientific project. Particular gratitude is extended to the Editor-in-Chief, Prof. Dr. Endre Boros, for his interest and confidence in, and support of our special issue from the very beginning of the project, to Managing Editor, Katie D'Agosta, for her continuous advice and guidance and her precious support in every respect, and to Ms. Anandhi Sankar, for her helpful service and positive attitude during the last two years. We thank all the authors for their great efforts and willingness to share their newest insights with the community, and we hope that their research will stimulate cooperation and progress at a worldwide and highest stage. 